\title{
NEW GENERALIZED 2D NONLINEAR INEQUALITIES AND APPLICATIONS IN FRACTIONAL DIFFERENTIAL-INTEGRAL EQUATIONS
}

\author{
BIN ZHENG
}

\begin{abstract}
In this paper, we study some new generalized 2D nonlinear Gronwall-Bellman type inequalities, which provide explicit bounds for unknown functions concerned, and are useful in the analysis of qualitative and quantitative properties for solutions to fractional differential and differential-integral equations. The presented inequalities are of new forms compared with the existing results so far in the literature. For illustrating the validity of the results presented, we present one application for them, in which the boundedness, uniqueness, and continuous dependence on the initial value and parameter for the solution to a certain fractional differentialintegral equation are investigated.
\end{abstract}

Mathematics subject classification (2010): 26D10.

Keywords and phrases: Gronwall-Bellman type inequality, fractional differential equation, fractional differential-integral equation, boundedness, qualitative analysis, quantitative analysis.

\section{REFERENCES}

[1] T. H. Gronwall, Note on the derivatives with respect to a parameter of solutions of a system of differential equations, Ann. of Math. 20 (1919), 292-296.

[2] R. Bellman, The stability of solutions of linear differential equations, Duke Math. J. 10 (1943), 643-647.

[3] H. X. Zhang, F. W. Meng, Integral inequalities in two independent variables for retarded Volterra equations, Appl. Math. Comput. 199 (2008), 90-98.

[4] B. G. PAChPATte, Inequalities for Differential and Integral Equations, Academic Press, New York, 1998.

[5] R. P. Agarwal, S. F. Deng, W. N. Zhang, Generalization of a retarded Gronwall-like inequality and its applications, Appl. Math. Comput. 165 (2005), 599-612.

[6] W. S. Cheung, J. L. Ren, Discrete non-linear inequalities and applications to boundary value problems, J. Math. Anal. Appl. 319 (2006), 708-724.

[7] W. N. Li, Some delay integral inequalities on time scales, Comput. Math. Appl. 59 (2010), 1929 1936.

[8] F. W. Meng, W. N. LI, On some newnonlinear discrete inequalities and their applications, J. Comput. Appl. Math. 158 (2003), 407-417.

[9] Q. H. MA, Estimates on some power nonlinear Volterra-Fredholm type discrete inequalities and their applications, J. Comput. Appl. Math. 233 (2010), 2170-2180.

[10] W. S. WANG, A class of retarded nonlinear integral inequalities and its application in nonlinear differential-integral equation, J. Inequal. Appl. 2012:154 (2012), 1-10.

[11] A. Gallo, A. M. Piccirillo, About some new generalizations of Bellman-Bihari results for integro-functional inequalities with discontinuous functions and applications, Nonlinear Anal. 71 (2009), e2276-e2287.

[12] O. Lipovan, Integral inequalities for retarded Volterra equations, J. Math. Anal. Appl. 322 (2006), 349-358.

[13] S. H. SAKER, Some nonlinear dynamic inequalities on time scales and applications, J. Math. Inequal. 4 (2010), 561-579. 
[14] S. H. SAKER, Some nonlinear dynamic inequalities on time scales, Math. Inequal. Appl. 14 (2011), 633-645.

[15] Y. G. Sun, On retarded integral inequalities and their applications, J. Math. Anal. Appl. 301 (2005), $265-275$.

[16] R. A. C. Ferreira, D. F. M. Torres, Generalized retarded integral inequalities, Appl. Math. Lett. 22 (2009), 876-881.

[17] F. W. Meng, W. N. LI, On some new integral inequalities and their applications, Appl. Math. Comput. 148 (2004), 381-392.

[18] Y. H. KIM, Gronwall, Bellman and Pachpatte type integral inequalities with applications, Nonlinear Anal. 71 (2009), e2641-e2656.

[19] W. N. Li, M. A. HAn, F. W. Meng, Some new delay integral inequalities and their applications, J. Comput. Appl. Math. 180 (2005), 191-200.

[20] H. X. Zhang, F. W. Meng, On certain integral inequalities in two independent variables for retarded equations, Appl. Math. Comput. 203 (2008), 608-616.

[21] B. G. PAChPATte, Explicit bounds on certain integral inequalities, J. Math. Anal. Appl. 267 (2002), 48-61.

[22] Z. L. YuAn, X. W. YuAn, F. W. Meng, Some new delay integral inequalities and their applications, Appl. Math. Comput. 208 (2009), 231-237.

[23] H. P. YE, J. M. GAO, Y. S. Ding, A generalized Gronwall inequality and ins application to a fractional differential equation, J. Math. Anal. Appl. 328 (2007), 1075-1081.

[24] F. C. JiAng, F. W. MENG, Explicit bounds on some new nonlinear integral inequality with delay, J. Comput. Appl. Math. 205 (2007), 479-486.

[25] F. W. Meng, Q. H. Feng, B. Zheng, Explicit Bounds to Some New Gronwall-Bellman-Type Delay Integral Inequalities in Two Independent Variables on Time Scales, J. Appl. Math. 2011 (2011), Article 754350, 1-25. 